\title{
KI-Systeme zu Lande, zu Wasser und in der Luft
}

\author{
Joachim Hertzberg
}

Online publiziert: 18. Februar 2011

(C) Springer-Verlag 2011

Liebe Leserin, lieber Leser!

Was, schon wieder ein KI-Heft über Robotik?! Da hat es doch erst Heft 3/2010 über SLAM und 4/2010 über Cognition for Technical Systems gegeben - wird das jetzt hier eine Robotik-Zeitschrift?

Tatsächlich wurde bei der Planung der Heftthemen dieser mögliche Eindruck erwogen. Ich habe viel Erfahrung mit solchen Reaktionen: Wer mich in meinem Büro besucht, kommt unweigerlich an Büro- und Laborräumen vorbei, in denen Roboter herumstehen oder -fahren, Laserscanner scannen und ein Greifarm greift, was bei Besuchern offenbar direkt zur Überzeugung führt: „,So, Sie machen also Robotik!“ Worauf ich inzwischen zu antworten pflege: „Wenn Ihr Gemeindepastor seine Predigt aufm PC tippt, würden Sie dann sagen, er macht Informatik?“

Von Zeit zu Zeit müssen wir uns in der KI daran erinnern, worum es eigentlich geht. Meine eigene Vorlesungsdefinition lautet: Die KI ist der Teil der Informatik, der mittels algorithmischer Modelle Leistungen des Denkens, Tuns und Wahrnehmens untersucht. Ich bin sicher, dass Sie nicht alle diese Sicht teilen: Manche werden sie zu stark, andere zu schwach finden; dem einen wird sie zu technisch sein, der anderen zu „kognitiv“ - darüber kann man ein andermal gern diskutieren. Im Moment kommt es mir darauf an, und vielleicht ist das konsensfähig: Sowohl (rationales) Denken als auch (physisches) Tun als auch (interpretierendes) Wahrnehmen gehören ,irgendwie“ mit dazu zur KI, oder?

Wenn das so ist, dann ist Arbeiten mit Robotern im Herzen in der KI - und die KI-Zeitschrift sollte das spiegeln.

J. Hertzberg $(\varangle)$

Osnabrück, Deutschland

e-mail: joachim.hertzberg@uos.de
Ist also KI gleich Robotik? Nein, natürlich nicht: Gehen Sie auf eine große Robotik-Konferenz wie die ICRA oder sehen Sie ins Springer Handbook of Robotics und staunen Sie, was es in der Robotik sonst noch an Mechatronik-, Sensorikund Algorithmik-Problemen gibt, von denen Sie in dieser Zeitschrift nie etwas lesen werden! Ist KI nur KI, wenn dabei ein Roboter vorkommt? Nein, natürlich auch nicht: Die klassischen KI-Themen KR, Lernen, Planen etc. etc. haben schon immer auch abseits von Robotern Sinn gehabt, und dabei bleibt es. (Die Themenvorschau am Anschluss an dieses Editorial zeigt Ihnen wie üblich, welche Themen nächstens in dieser Zeitschrift zu erwarten sind, und zufällig ist da nichts mit Robotik dabei.)

In den frühen Jahren scheint es normal gewesen zu sein, Fortschritt an „KI-Systemen“ zu zeigen: an integrierten Systemen, die in der Regel mehr als eines der KI-Themen berührten. Klassiker wie SHRDLU, GPS, DENDRAL, oder, um im Lande zu bleiben, NAOS waren Beispiele für solche integrierten Systeme - und natürlich der Roboter SHAKEY. Doch Forschung an und mit integrierten Systemen voran zu treiben, hatte eine negative Folge: Methodischer Fortschritt, an einem kompletten KI-System erzielt, ist außerhalb des Systems schwer nachzuvollziehen und oft unmöglich zu reproduzieren. Es war daher in den 1980er Jahren ein wichtiger methodischer Schritt für die KI, die unterliegenden Probleme durch Abstraktion und Formalisierung aus dem Korsett ihrer Systemkontexte herauszulösen und damit Fortschritt in KI mit methodischer Strenge zu erlauben, statt nur mit „Magie“.

Wenn auch diese Abstraktion und Formalisierung notwendig waren für die Entwicklung des Gebiets, so haben sie ihren Preis: Die KI-Themen Lernen, Planen etc. können anschließend, wenn man es denn will, auch ausschließlich abstrakt und formal bearbeitet werden - ohne Bezug zu ihrer Einbettung in ein übergeordnetes System, ohne den Kontext 
anderer KI-Themen, ohne Anschluss zum Rest der KI. Da ist sie, die Zersplitterung und Zerbröselung der KI, die wir zuweilen beklagen, wenn wir auf Konferenzen feststellen, es gebe heute ,irgendwie keinen Zusammenhalt mehr“ über die KI-Themen hinweg. Ja, wir haben hart dran gearbeitet, dass diese Themen auf eigenen Füßen stehen können, klar definierte Probleme bearbeiten und Fortschritt ohne Rückgriff auf irgendein wolkiges Konzept von Intelligenz beurteilen lassen. Auf eigenen Füßen stehen sie nun, freuen wir uns dran!

Doch erinnern wir uns gelegentlich: Es ging an sich nicht um Lernen, Planen etc. - es ging um KI! Um clevere Systeme, die in anspruchsvollen Umgebungen zu Lande, zu Wasser und in der Luft denken, tun und wahrnehmen können, und zwar alles das! Es ging um ... nein, nicht Roboter, sondern um integrierte Systeme - doch wenn die auch tun und wahrnehmen sollen, helfen Aktuatoren und Sensoren wie in der Robotik ganz ungemein! Ich wünsche Ihnen also anregende Lesestunden mit den hier zusammengestellten Arbeiten zu Offroad-KI-Systemen! Und ich verspreche, dass es auch in Zukunft Themenhefte dieser Zeitschrift zu physischen KI-Systemen geben wird. Sie dürfen sie gern Roboter nennen!

Freundliche Grüße,

\section{Joachim Hertzberg}

\section{Themenvorschau}

\section{Schwerpunkt - Emotion and Computing}

How does emotion and computing work together? Can a computer be emotional? Can a computer system recognize, understand and react on human emotions? In recent years computer science research has dealt with these questions in numerous projects. Several approaches have been made concerning emotion recognition, emotion modeling, generation of emotional user interfaces and dialogue systems as well as anthropomorphic communication agents. Regarding human behaviour, emotions play an essential role in decision making, as well as in perception and learning. Modern media take an increasing role regarding human social interaction. Human computer interfaces integrate the recognition and synthesis of nonverbal parts of communication.

This special issue of KI focusses on the role of artificial intelligence in emotional computer systems. This includes computational models of emotion, their applications and the methods of artificial intelligence applied. More detailed information can be found on http://www.emotion-andcomputing.de/.
Interested authors are asked to contact the guest editor as soon as possible:

\section{Prof. Dr. Dirk Reichardt}

Prorektor und Dekan Fakultät Technik

Professor für Informatik

Duale Hochschule Baden-Württemberg Stuttgart

Jägerstraße 56

70174 Stuttgart, Deutschland

reichardt@dhbw-stuttgart.de

\section{Schwerpunkt - 25 Jahre KI und KI-Zeitschrift}

Seit 1987 erscheint die Zeitschrift „Künstliche Intelligenz“ in einem Verlag, der Vorgänger „KI-Rundbrief“ wurde als Manuskript von Universitäten bzw. Forschungseinrichtungen kopiert und verteilt. In diesem ,Jubiläumsheft“ wird unter verschiedenen Aspekten auf diese 25 Jahre KI und KIZeitschrift zurückgeblickt.

Darüber hinaus ist geplant, auch die nähere und fernere Zukunft der KI und der Zeitschrift zu beleuchten.

Interessierte Autorinnen und Autoren werden gebeten, sich möglichst bald an den Gastherausgeber zu wenden. Es sind zur Vergangenheit und Zukunft der KI und ihrer Zeitschrift Beiträge aller Art erwünscht!

\section{Dr. Andreas Günter}

HITeC e.V./Uni Hamburg

Vogt-Kölln-Str. 30

22527 Hamburg, Deutschland

Tel: 040/42883-2456

guenter@informatik.uni-hamburg.de

\section{Schwerpunkt - KI Sprachen (AI Languages)}

In the past, mainly Prolog and Lisp were considered as the most important representatives of artificial intelligence programming languages. However, in the meantime, the range of such languages has been extended by the development of new areas of interest within AI. Existing languages have been extended, e.g. by constraints, and there are developments of multi-paradigm languages which support more than one programming paradigm and style with the aim of an efficient modeling and problem solving. But, furthermore, there are new directions of research and application, like languages of the Semantic Web, languages for planning and agent-oriented programming.

This special issue includes technical papers, reports on current research projects, interviews, summaries of doctoral theses, book reviews and others with focus on current developments and perspectives of languages in the area of AI. Topics of interest include but are not limited to: 
- Classical and new paradigms of AI programming

- Functional-logic, constraint-based, and further multiparadigm languages

- Inductive programming

- Languages of the Semantic Web

- Domain specific languages which support programming of AI-specific applications, e.g., planning applications and agent-oriented programming

Interested authors should contact the guest editor as soon as possible:

\section{Prof. Dr. Petra Hofstedt}

Brandenburgische Technische Universität Cottbus

Fakultät 1, Institut für Informatik

Lehrstuhl Programmiersprachen und Compilerbau

Postfach 101344

03013 Cottbus, Deutschland

hofstedt@informatik.tu-cottbus.de

\section{Schwerpunkt - Human-Computer Interaction (HCI)}

Bei fast allen computertechnischen Anwendungen spielt der Mensch eine zentrale Rolle. Aus forschungstechnischer Sicht hat dies eine Vielzahl von Auswirkungen: Um Konzepte und Entwicklungen besser auf den Menschen zuzuschneiden, sind vor und während der Konzeption und Umsetzung eines Projektes aufwendige Benutzerstudien und Evaluierungen durchzuführen. Hierzu zählen beispielsweise die Durchführung von Interviews mit potentiellen Nutzern und die Diskussion von Ergebnissen in Focus Groups.
Neben diesen Themen fallen in das Feld der HCI auch die Forschungsarbeiten im Bereich der Interaktion. Da visuelle Darstellungen nicht nur als eine reine Ausgabe von Ergebnissen am Ende einer langen Kette zu verstehen ist, sondern als fortlaufendes Kontrollinstrument, welches die gesamte Prozesskette begleitet, stellt hier die Interaktion ein äußerst wichtiges Element dar. Ziel ist es, die Barriere zwischen Mensch und Maschine möglichst effektiv und effizient zu überwinden, indem dem Benutzer eine möglichst intuitive Interaktion mit dem System angeboten wird.

Im Rahmen des Schwerpunkts Human-Computer Interaction werden Beiträge zu folgenden

- Bereichen vorgestellt:

- Psychologie und kognitive Wissenschaften

- Evaluierungstechniken

- Moderne Visualisierungs- und Interaktionsmetaphern

- Anwendungsprojekte

Interessierte Autorinnen und Autoren werden gebeten, sich an den Gastherausgeber zu wenden:

\section{Prof. Dr. Achim Ebert}

AG Computergrafik und HCI

Fachbereich Informatik

Technische Universität Kaiserslautern

Postfach 3049

67653 Kaiserslautern, Deutschland

Email: ebert@cs.uni-kl.de 